\title{
e.2e cONTRIBUCIONES FEMINISTAS A www.aibr.org PROBLEMAS EPISTEMOLÓGICOS DE LA DISCIPLINA ANTROPOLÓGICA: REPRESENTACIÓN Y RELACIONES DE PODER
}

\section{Carmen Gregorio Gil}

Profesora Titular. Departamento de Antropología Social. Universidad de Granada. Dirección: E. U. de Trabajo social. C/ Rector López Argüeta s/n 18071.Granada (España). E-mail: carmengg@ugr.es.

\begin{abstract}
Resumen
Palabras clave

Antropología feminista, género, etnografía.
\end{abstract}

Este trabajo se propone destacar las aportaciones desde enfoques feministas a un debate epistemológico central en la disciplina antropológica: Las relaciones de poder implicadas en la representación de la otredad y en la producción de conocimiento científico.

\begin{abstract}
This work focuses on the epistemological contributions of feminist approaches to a central debate in social anthropology: the gender-power relations involved in the representation of Otherness as well as in the production of knowledge.
\end{abstract}

\section{Keywords}

Feminist anthropology, gender, ethnography.

Co omo señala DiLeonardo a comienzos de la década de los 1990 la investigación antropológica de orientación feminista "has come of age", ha cumplido la mayoría de edad, (1991: 1) y se dispone de un importante corpus teórico y epistemológico en diferentes campos de la antropología social, pero también en disciplinas afines ${ }^{1}$ y dentro de los estudios denominados feministas ${ }^{2}$. Sin embargo, a pesar de la vasta producción teórica y etnográfica su contribución en el desarrollo de la antropología social no ha sido lo suficientemente reconocida. El título "A divided population: what is good for some

\footnotetext{
${ }^{1}$ Véase en el trabajo de Stacey y Thorne (1985) las aportaciones de la antropología feminista en su contribución al avance de la teoría social.

${ }^{2}$ Como señalan para el caso del Estado español Ballarín, Gallego y Benlloch (1995). Véase también Maquieira, Gómez-Ferrer, y Ortega (1989) con quién estamos de acuerdo cuando plantea en relación a las aportaciones de la antropología social en el desarrollo del pensamiento occidental feminista, que ésta no podría estar ausente como consecuencia del: "[...] carácter globalizador de la antropología social en el análisis de la experiencia humana y sus diversas realizaciones en el tiempo y en el espacio, así como su preocupación tradicional por dilucidar los modos de representación simbólicos e ideológicos" (Maquieira et a., 1989: 9).
} 
is irrelevant for others" (del Valle 1993b: 1-5) con el que Teresa del Valle enuncia uno de los apartados de su introducción al libro Gendered Anthropology condensa la paradoja acerca de las aportaciones feministas en antropología, puesto que, lo que ha sido resaltado con fuerza como un enriquecimiento para la antropología social por muchas autoras y menos autores, incluso, lo que le otorga "credibilidad social" (del Valle 2000b: 10), es visto como un problema para otros.

La pregunta que nos hacemos con Strathern (1987a) es si el énfasis en las diferencias constituyen bases comunes para la mutua convergencia entre antropología y feminismo, ¿por qué esa resistencia? o ¿por qué su ignorancia, si sus preocupaciones entran de lleno en el debate antropológico acerca de la conceptualización de cultura y los problemas epistemológicos de representación?

La perspectiva feminista en la teoría antropológica y su institucionalización, como señalan Mukhopadhyay y Higgins "afectó a la antropología desafiando principios y teorías así como los métodos de la etnografía tradicional" (1988: 461; traducción mía). Sin embargo, este desafío no siempre ha supuesto una transformación de la teoría antropológica. Como nos plantea Stolcke:

\footnotetext{
No se ha logrado, sin embargo una transformación de la mirada antropológica en general. Está más que demostrado que los sistemas de género constituyen uno de los principios de estructuración social fundamentales, de modo que tal revisión etnográfica y teoría radical es imperativa si se pretende comprender de modo cabal lo que la antropología ha entendido desde siempre como su problema característico, a saber, la unidad humana en la diversidad cultural. Esa reticencia puede parecer paradójica a la vista de lo relativo de la antropología en general y del impacto, aunque muchas veces no reconocido, que la antropología feminista ha tenido al demostrar la producción de las diferencias (Stolcke, 1996: 342-343).
}

Las contribuciones feministas a los debates epistemológicos de la disciplina antropológica entroncan con el cuestionamiento de la supuesta "objetividad" del conocimiento científico y la "crisis de representación" que ocupan a la antropología social desde el periodo denominado postcolonial. Si bien, como han analizado diferentes autoras ${ }^{3}$, la corriente postmoderna en antropología rechaza o infravalora las contribuciones que desde las perspectivas feministas se han venido realizando acerca de las relaciones de poder implicadas en el método etnográfico, las nociones de "otredad" e identidad en el proceso de trabajo de campo, así como las propuestas de reflexividad y de escritura etnográfica experimentales.

Intentaré reflejar en este artículo las aportaciones feministas más significativas, a mi juicio, relativas a los problemas de representación de ese "otro" encarnado en un cuerpo que deviene mujer y acerca de la "generización" del conocimiento producido en interacción con otros sujetos en el proceso etnográfico a lo largo del saber acumulado, fundamentalmente por las antropólogas, desde los años 30 del pasado siglo.

\footnotetext{
${ }^{3}$ Entre otras, Bell, Caplan y Wazir Jahan (1993), Di Leonardo (1991a), Mascia-Lees, Sharpe y Ballerino (1989), Morgen (1989), Okely (1992), Okely y Callaway (1992), Stolcke (1993), Strathern (1987b, 1987c).
} 


\section{"Mujeres etnógrafas" y sujetos "generizados": Reescribir las etnografías.}

A comienzo de los años 1970, ante el reconocimiento de que los fines que habían guiado el desarrollo de la disciplina antropológica no podían separarse de los procesos políticos de dominación y colonización, se asiste a lo que se ha convenido en llamar la "crisis de la antropología" (Hymes, 1972; Stocking, 1987) ${ }^{4}$. Se derrumbaba el ideal de la consolidación de una "Ciencia libre de valores" para reconocer que los conocimientos de otras culturas que proporcionaba la antropología al emerger en un contexto histórico y político desigual contribuían a consolidar la subordinación, de los "otros culturales"-sujetos, pueblos, comunidades o naciones ${ }^{5}$.

Este asunto no era ajeno a la antropología feminista que en sus primeros cuestionamientos incidió en problematizar sobre cómo desde el conocimiento antropológico se representaba a las mujeres - a ese "otro"- a partir del proceso de trabajo de campo etnográfico. Lo que se configuró como "antropología de la mujer" en su objetivo de visibilizar y transformar el papel que se otorgaba a las mujeres en las sociedades que se estudiaban desde la antropología puso de manifiesto el androcentrismo o viricentrismo ${ }^{6}$ como una forma más de conocimiento producido desde una mirada particular $^{7}$ que contribuía reproducir desigualdades de género. La vida de las mujeres de muchas sociedades no era analizada con el mismo protagonismo y minuciosidad que la de los hombres, bien porque se partía de su papel secundario en la sociedad y se devaluaban sus prácticas e interpretaciones, bien porque las sociedades estudiadas estaban estructuradas por relaciones de dominación de género que ocultaban o deslegitimaban las contribuciones de las mujeres. La elección de informantes clave -casi siempre varones- como representantes significativos de la cultura invisibilizaba otras muchas voces dentro de sociedades jerarquizadas.

Desde la antropología social se habían reconocido las limitaciones del antropólogo -hombre- de acceder a las actividades e interpretaciones de las mujeres sobre todo en sociedades muy segmentadas en términos de género ${ }^{8}$. Pero ello no cuestionaba el conocimiento que se estaba produciendo, dado que se justificaba como una carencia sin más. Un recurso metodológico muy utilizado para corregir lo que era considerado como "desviación" o "sesgo" era la realización del trabajo de campo en pareja matrimonial ${ }^{9}$. En este equipo se daba una suerte de especialización en la que la esposa se ocupaba de las mujeres y el esposo del "conjunto de la sociedad". Las mujeres en el

\footnotetext{
${ }^{4}$ Acerca de la alianza de la antropología con el colonialismo véanse los trabajos de Asad (1973), Huizer y Mannheim (1979). Sobre los planteamientos epistemológicos más concretamente Hacking (1983), Salmond (1982), Wolfram (1982)

${ }^{5}$ Véase Wolf (1982) para el análisis histórico y económico político en antropología social. Igualmente la propuesta de Stolcke (1993) acerca de la utilidad de llevar a cabo una "etnografía de la historia de la antropología" para desvelar estas relaciones de poder en la representación del "otro".

El término androcentrismo es el más utilizado en la literatura feminista antropológica para referirse a la forma de producir conocimiento desde valores hegemónicos masculinos o posiciones autocentradas en el hombre como eje de la vida social. Schrijers a diferencia del término de origen americano androcentrismo, utiliza el termino viricentrismo (1979: 97).

${ }^{7}$ Otras serían el etnocentrismo, racismo o clasismo.

${ }^{8}$ Véase Golde (1970).

${ }^{9}$ Conocidos matrimonios que han trabajado conjuntamente en el campo y en los que se ha observado una suerte de especialización en términos de género en sus trabajos son los Bohannan, Dwyer, Fernea, Hart, Smith o los Wolf. Ellas escribieron, Return to Laughter (Laura Bohannan, bajo el seudónimo E.S. Bowen, 1954); Images and Self Images: Male and Female in Marocco (Daisey H. Dwyer, 1978), Guest of the Sheik (Elizabeth W. Fernea, 1969) Tras la puerta del patio. La vida cotidiana de las mujeres rifeñas (Ursula K. Hart, (1998), Baba of Karo (Mary Smith, 1954), The House of Lim (Margery Wolf, 1968).
} 
campo se encargaban de ese "otro específico", que era interpretado por la corriente general de la antropología incluso con mayores tintes de exotismo ${ }^{10}$ o misterio y que venía a complementar la totalidad de las prácticas y discursos que representaban los hombres de las sociedades estudiadas por los antropólogos.

La vida del "otro femenino" constituía un apartado más de las culturas estudiadas por la antropología que venía a sumarse al resto de los aspectos considerados centrales en la producción social, en los que las mujeres participaban sólo como objetos. Scheper-Hughes (1983) cita cómo Ardener (1975a) observó que Evans-Pritchard trató a las mujeres con la misma tosquedad que trató al ganado, esto es, "omnipresentes pero mudas con respecto a su habilidad para mostrarnos los significados de la cultura y las relaciones sociales nuer" (Scheper-Hughes, 1983: 110). Otras autoras como Provansal (1999: 55) han mostrado cómo la incidencia de las mujeres en la vida social se oculta en "la mente del etnógrafo" para quién la iniciativa y trasgresión femenina es una posibilidad remota.

Los ejemplos de los sesgos androcéntricos en la literatura etnográfica que proyectan un orden dado en las relaciones de género podrían repetirse hasta la saciedad. Es por ello que, desde la crítica feminista en antropología, se planteará la necesidad de reescribir las etnografías con una visión menos androcéntrica. Será necesario compilar más datos sobre la vida de las mujeres ${ }^{11}$ y/o desmenuzar las etnografías realizadas por los antropólogos ${ }^{12}$ para desvelar sus lagunas o interpretaciones fundamentadas en preconcepciones o estereotipos de género.

Se obtuvieron cantidades ingentes de datos etnográficos, que complementaban o contradecían aspectos de la vida y representaciones de las sociedades estudiadas. El problema de fondo se encontraba en el lugar que otorgaba a las mujeres la teoría antropológica, en definitiva en una cuestión de representatividad, pues, como señala Edwin Ardener, "En la fase de 'observación' de los trabajos de campo, el comportamiento de la mujer se ha estudiado, por su puesto, al igual que el del hombre, de forma exhaustiva: sus matrimonios, su actividad económica y ritos y todo lo demás" (Moore, 1991: 13).

La identificación del androcentrismo en la literatura antropológica y los diferentes niveles en el que este se manifestaba ${ }^{13}$ produjo una serie de propuestas teóricas y metodológicas de gran interés, en tanto que ponían de manifiesto que los modelos de dominación de género del propio contexto sociocultural del que formaban parte los y las investigadoras, se reflejaban en los marcos interpretativos. Se entraba de lleno en el debate antropológico acerca de la alteridad y las relaciones de dominación que implica el proceso de dar cuenta de la diversidad, de "lo otro" a partir del nosotros mismos.

\footnotetext{
${ }^{10}$ Véase, por ejemplo, en Provansal (1999) cómo el discurso colonial sobre el Magreb potenció una representación de las mujeres en las que el exotismo y erotismo eran notas reinantes. El exotismo en las mujeres convertido en erotismo respondía al peso del discurso masculino occidental en la representación de las "otras".

${ }_{11}$ Un ejemplo de ello es el trabajo de Weiner (1976) que escribirá una etnografía sobre los Trobriand ofreciendo una visión de las mujeres bien diferente a la de Malinowski

12 Di Leonardo (1979), por ejemplo, se centrará su trabajo en revisar los trabajos etnográficos de Goodenough.

${ }^{13}$ Véase una descripción detallada en Moore (1991) o en Scheper-Hughes (1983).
} 
El descubrimiento de diferentes formas de manifestación de sesgos de carácter androcéntrico en el proceso de construcción teórica llevó a Edwin Ardener (1975) a proponer la teoría de los "grupos silenciados". Esta teoría implicaba reconocer la existencia de relaciones de poder que posibilitan a los grupos dominantes una mayor capacidad de generar y controlar las formas de expresión, quedando los grupos dominados silenciados o en la obligación de recurrir a las ideologías dominantes para expresarse. Como consecuencia se hacía necesario tomar en consideración la existencia de discursos hegemónicos que dejaban fuera otras formas de interpretar la realidad, o incluso la imposibilidad de acceder a estos grupos "subalternos" o subculturas -en tanto que en el marco de relaciones de dominación sólo podían "romper su silencio" adoptando modelos de expresión hegemónicos. Mathieu (1978) añadirá un matiz, relacionado con las prácticas de investigación: no es que las mujeres sean mudas, lo que ocurre es que no son interrogadas ni tenidas en cuenta para algunas cuestiones.

Una de las estrategias metodológicas derivadas de la propuesta de Ardener (1975a) fue partir de la supuesta "identidad femenina" para poder acceder a las elaboraciones de las mujeres de otras culturas. Se plantea que el género del antropólogo, hombre, condiciona los datos y limita la información que se obtiene de las mujeres. Las antropólogas de esta forma son convertidas entonces en sujetos de conocimiento válidos para los grupos femeninos dominados -que serán convertidos en "objeto de conocimiento". Esta propuesta partía de la categoría homogenizadora "mujer" y pasaba por alto la confrontación etnográfica que se da en el trabajo de campo ${ }^{14}$. Además, aunque fuesen mujeres antropólogas las que interactuaban con otras mujeres no tenían por qué estar a salvo de la reproducción de las estrategias de investigación dominante. Como, por ejemplo, pusieron de manifiesto Okely (1992) y Atkinson (1982) al referirse a la objetualización que tiene lugar en la realización de algunas biografías de mujeres por antropólogas.

En definitiva, no podía sostenerse que para conocer a un grupo -i.e., mujeres- fuese necesario pertenecer a él -i.e., ser mujer. Además del esencialismo, naturalización y reificación que entraña teóricamente la categoría género, este tipo de empatía femenina o "feminidad universal" (Strathern, 1981) parecía renunciar a la operación de extrañamiento necesaria en la empresa antropológica.

Esta propuesta se situaba todavía dentro de la autoridad del método científico, que en su búsqueda de la "objetividad", trataba de disminuir los "sesgos personales". Tomaba relación, en parte, con el asunto de la "variable personal" o "ecuación personal"15 del/de la antropólogo/a en el campo que venía preocupando a la antropología social. El sexo del/de la antropólogo/a era considerado en la metodología de investigación etnográfica como una variable más -otras eran, edad, color de piel, estado civil- que influía en la obtención de los datos. En esta línea, diferentes trabajos se centraban en analizar qué ventajas e inconvenientes suponía ser hombre o mujer en el campo, así como otra serie de especificaciones (Gregory, 1984; Kessing, 1985; Whitehead y Conaway, 1986).

\footnotetext{
${ }^{14}$ Véase en Shapiro (1981) los cuestionamientos a la propuesta acerca de que las mujeres son las que deben estudiar a otras mujeres en el trabajo de campo.

${ }^{15}$ Véase López Coria (1991).
} 
Pero, como plantea Bell (1993), el principal problema de estos trabajos, cuyas conclusiones se muestran contradictorias y problemáticas, es que se analizan las dificultades o ventajas en el campo desde la perspectiva de lo "femenino" versus lo "masculino". La atribución de lo "masculino" y lo "femenino" a cuerpos diferenciados sexualmente como hombre o mujer antropólogos, además del esencialismo que entraña, implica partir de rígidos planteamientos positivistas que esquematizan el juego de identidades implicado en la interacción social.

Sin embargo, este análisis posibilitó que las experiencias de las mujeres en el campo fuesen contrastadas. El "ser mujer" en el campo mostraba una enorme variabilidad y comenzó a considerarse todo un elenco de diferencias en función del sexo, género, edad, etnicidad o religión (Ardener, 1984; Warren, 1988). Estas diferenciaciones sociales vendrán a añadirse a la categoría género contribuyendo a deconstruir la presupuesta universalidad de la identidad femenina.

Las propuestas metodológicas desde la crítica feminista se dirigirán a hacer visibles las elaboraciones de los sujetos, su acción en la vida cotidiana en interacción con los sectores dominantes, huyendo de cualquier tipo de esencialismo y cosificación que "busque la especificidad femenina" o "esencialice su especificidad" (Juliano, 1990). A juicio de esta autora, "[e]l objetivo de nuestras investigaciones puede ceñirse al ámbito más modesto pero también más significativo de rastrear, describir y hacer evidentes las propuestas que al respecto han ido elaborando las mujeres -aisladamente o en conjunto- en su cotidiana interacción con los sectores dominantes" (Juliano, 1990: 13).

Se opera por tanto la deconstrucción de la "identidad femenina" desde la propuesta de una noción de género más relacional y desde la propia práctica etnográfica, que se inclinará por un camino más dialógico, al reconocer las relaciones de poder fluidas y cambiantes que se dan en el campo y la diversidad de posiciones de los sujetos implicados.

Si bien, ante la observación de la frecuencia de las monografías sobre mujeres escritas por mujeres desde la temprana etnografía ${ }^{16}$, me parece interesante, en la discusión acerca de la representación del "otro", preguntarnos con Callaway (1992). ¿por qué las mujeres antropólogas han creado sujetos generizados, algo que por el contrario no parecen haber hecho los antropólogos hombres? Callaway relaciona este hecho con la construcción de la masculinidad del antropólogo, que lo sitúa en la concepción de 'el Hombre' como representativo de la sociedad total.

\section{El desafío a la objetividad y el desenmascaramiento de las relaciones de poder: el "conocimiento situado".}

En el afán de ofrecer datos sobre la realidad de las mujeres en las diferentes sociedades, se puso de manifiesto que el género/sexo del antropólogo/a influía en la visión que se daba de una determinada

\footnotetext{
${ }^{16}$ Constituyen ejemplos de ello Talbot (1968), Leith-Ross (1965), Kaberry (1939, 1952), Landes (1971, 1979) y Underhill (1936).
} 
sociedad. Estos hallazgos supusieron un desafío para la objetividad que presidía el conocimiento etnográfico, al mismo tiempo que desenmascaraba las relaciones de poder en la representación del "otro", en este caso las mujeres. El discurso colonial hacia las mujeres se manifestaba con tintes diferenciales al que representaba a los hombres ${ }^{17}$.

La conceptualización del género como sistema de relaciones de poder hará que las propuestas que se desarrollen vayan más allá del reconocimiento de la dificultad de acceder a la información sobre las prácticas e interpretaciones de las mujeres por el hecho de ser antropólogo hombre o mujer. Dificultad, por otro lado, que no tambaleaba los presupuestos teóricos y metodológicos, bien asentados, en la disciplina antropológica.

El género se convertirá en una categoría analítica central tanto en la práctica de la etnografía como en los marcos teóricos de la disciplina. Ello conducirá a cuestionar la pretendida neutralidad del conocimiento en tanto que afectado por manifestaciones particulares de dominación -androcentrismo y heterosexismo- que son transferidas a las prácticas de investigación. Hasta el momento no se había puesto en cuestión la objetividad científica como logro a obtener, sí la necesidad de llenar lagunas, incluir las aportaciones "femeninas", en definitiva devolver al conocimiento su "objetividad". Pero desde la crítica feminista -en donde tendrán un papel protagonista las epistemólogas de las ciencias naturales ${ }^{18}$ - se señalará que el saber científico se construye en un espacio y tiempo determinado y, como consecuencia de su legitimidad para conceptualizar de forma universal la verdad y lo significativo, contribuye a justificar relaciones de poder.

Los cuestionamientos de la epistemología feminista implican la relativización de la razón, puesto que se apoyan en que, el conocimiento de diferentes experiencias da lugar a distintos conocimientos. Se resaltará, por tanto, el carácter parcial, histórico y no universal del conocimiento e incluso de la misma razón ${ }^{19}$. Al cuestionar supuestos dados por sentados acerca de la verdad, el poder, el individuo o el lenguaje se desvelará cómo el saber científico puede convertirse en un instrumento de legitimación de la cultura occidental.

Todo ello llevará a calificar desde las perspectivas feministas como "mala ciencia" aquella que legitima biológicamente la desigualdad sexual (Laslett, Gregory, Longino y Hammonds, 1996: 2). La antropología feminista observará en la ciencia su papel prioritario en la creación de subjetividades, identidades y sexualidades ${ }^{20}$, lo que le llevará a disolver la relación esencial entre cuerpo y género en su papel legitimador de desigualdades.

\footnotetext{
${ }^{17}$ Véase Rohrlich-Leavitt, Sykes y Weatherford (1975), Leacock (1979), Schrijvers (1979).

${ }_{18}$ Juliano (2000: 31) situará a partir de las obras de las epistemólogas de las Ciencias Naturales Hilary Rose $(1983,1987)$ y Evelyn Fox Keller $(1983,1989,1991)$, un giro en la concepción de la ciencia. Puede verse una revisión en castellano de las aportaciones feministas al cuestionamiento del saber científico, en especial a las ciencias biomédicas, en Ortiz (1999).

${ }^{19}$ Véase Flax (1987), Haraway (1991) Harding y O'Barr (1987), Keller (1985), Laslett (1990), Merchant (1980).

${ }^{20}$ Véanse en esta dirección los trabajos de Martín (1996) y Rapp (1999).
} 
La deconstrucción operada en el concepto de género afectará a la noción de "identidad de género" en el proceso etnográfico, señalando la importancia de la dialógica discursiva en la construcción de las diferentes representaciones (Flax, 1987). Por su lado, la necesidad de producir un conocimiento "situado" llevará a hablar de "objetividades encarnadas" (Haraway, 1988), reconociendo los aspectos subjetivos implicados en el proceso etnográfico.

\section{Epistemología feminista y etnografía postmoderna: Un proyecto de conocimiento "generizado"}

La antropología feminista se anticipó al giro postmoderno (Laslett et al., 1996). Pero, a pesar de los cuestionamientos que planteó acerca de los problemas de representación y sus propuestas metodológicas, desde el "academicismo etnográfico postmoderno"21 no fueron tenidas en cuenta, alegando su vinculación política y teórica con el "Feminismo". Nos preguntamos con Bell (1993), Caplan (1993), di Leonardo (1991), Morgen (1989), Mascia-Less, Sharpe y Ballerino (1989) a partir de las críticas a la antropología feminista que plantean los trabajos de Clifford y Marcus (1986) ${ }^{22}$, Marcus y Fischer (1986) o Rabinow (1986) ¿cómo aceptar las críticas que eluden o rechazan la contingencia histórica de mentalidades sexistas o androcéntricas, cuando sus propuestas sin embargo reconocen, la contingencia histórica de las mentalidades racistas y colonialistas? ¿cómo interpretar por ejemplo el 'cosmopolismo crítico' propuesto por Rabinow (1986) para no esencializar la diferencia, y asumir el esencialismo que entrañan sus argumentos en su crítica al discurso feminista y del tercer mundo? ¿No será que el "sexo/género" de los sujetos de conocimiento es utilizado como forma de invalidar las propuestas desde el feminismo?

Es práctica habitual criticar a las antropólogas por la elección de un sujeto implicado en su propia experiencia en vez de reconocer, como indica del Valle (1993b), sus formas de encarar "con creatividad el dilema dentro-fuera" o "situarse en la contradicción de reconocer la diversidad a través de las mujeres, mientras se reconoce la unidad" (Mascia-Lees et al. 1989), o las estrategias para manejar la confrontación ética y política al deconstruir las categorías sustanciales a su propio análisis -mujer, feminismo, opresión- al mismo tiempo que mantienen los objetivos políticos de contribuir al análisis de la desigualdad. En definitiva, su capacidad para reclamar la objetivación de su empresa, al mismo tiempo que reconocen la parcialidad de las verdades.

Mascia-Less, Sharpe y Ballerino (1989) son explícitas en su crítica a cómo la nueva etnografía postmoderna ignora las propuestas feministas:

Sin embargo los que aparecen como nuevos y excitantes hallazgos de esta nueva antropología posmodernista -que la cultura está compuesta de códigos de significados seriamente contestados, que el lenguaje y la políticas son inseparables, y que, al construir al "otro" se entablan relaciones de dominación- son hallazgos que han recibido

\footnotetext{
${ }^{21}$ Utilizo a sugerencia del profesor Gunther Dietz "academicismo etnográfico" en vez de "liberalismo etnográfico" originario de la tradición anglófona.

${ }^{22}$ Véase Gordon (1988) para una crítica feminista más exhaustiva al trabajo de estos autores.
} 
repetidas y enriquecedoras exploraciones en la teoría feminista en los pasados cuarenta años (Mascia-Less, Sharpe y Ballerino, 1989:52).

[...] Como recuerdan estas autoras "La teoría feminista. incluso en los 70, estaba comprometida no sólo con la comprensión de la experiencia de las mujeres de su otredad, sino también con la inscripción de las mujeres como otras en el lenguaje y en el discurso (Mascia-Less, Sharpe y Ballerino, 1989:53).

Las autoras ponen de manifiesto sus dudas ante una nueva autoridad constituida textualmente ${ }^{23} \mathrm{y}$ ante el peligro que encierra pretender que se están transformando las relaciones de poder en la disciplina, estableciendo un nuevo territorio de supremacía intelectual en la academia, una nueva "Verdad" 24 .

Aunque se atribuirá a los antropólogos anteriormente citados haber teorizado sobre los problemas de representación como se deduce de lo expuesto, esta crisis ya era evidenciada desde los planteamientos feministas: la representación de la realidad se alejaba de ser la imagen proyectada en un espejo para ser una realidad parcial y posicionada. Las propuestas feministas se diferencian de la etnografía postmoderna en que enraízan el conocimiento en las políticas y relaciones de desigualdad y no olvidan las condiciones materiales de existencia al formular sus teorías desde la práctica (Bell, Caplan y Karim 1993; DiLeonardo, 1991; Mascia-Lees et al., 1989).

Como señalan Mascia-Lees et al. (1989) la etnografía postmoderna no constituye una vía válida para la lucha por la exclusión y el cambio, al ignorar y oscurecer la explotación y las diferencias, con ello "la historia del colono puede ser leída independientemente del colonizado" (Mascia-Lees et al., 1989: 70). A juicio de Stolcke: "la 'nueva etnografía' pos-moderna pone de manifiesto su propio sentido de fragmentación y desaliento mirándose a sí misma. Al proclamar su propia carencia de autoridad niega, sin embargo, también a otros/as diversas la condición de sujetos dotados de poder de opinar" (1993: 173).

Otra de las propuestas metodológicas desde la crítica feminista relacionada con los problemas de representación dimana de su forma de entender la identidad de género en el proceso de investigación etnográfico. La antropología feminista se pregunta acerca de las implicaciones del antropólogo como conocedor generizado. $Y$ en sus propuestas plantea la disolución entre sujeto -conocedor- y objeto de conocimiento-, entre el yo y el otro para tomar un camino dialógico e intersubjetivo desde posiciones políticas situadas.

Las perspectivas feministas, al interpretar el propio acto de categorizar como una expresión de poder, se inscriben en una nueva etnografía que propone la deconstrucción de categorías fijas e inmutables asignadas a los sujetos. Sus planteamientos deconstruyen la noción de identidad de género como entidad fija que actúa en el campo, para proponer la noción de persona que experimenta y se

\footnotetext{
${ }^{23}$ Remiten al trabajo de Sangren (1988).

24 Aunque citan algunas excepciones como el trabajo de Owens "The Discourse of Others; Feminists and Postmodernism (Mascia-Lees et al., 1989: 55).

(C) Carmen Gregorio Gil. Publicado en AIBR. Revista de Antropología Iberoamericana, Ed. Electrónica 
transforma, en las relaciones con los otros (Strathern, 1988). De esta forma transciende la noción de persona unitaria dualizada, fijada como mujer o hombre. Desde este punto de vista conceptualiza la identidad en el proceso de interacción etnográfico como múltiple, contestada al tiempo que contradictoria (de Lauretis, 1986).

Plantea la comprensión de las múltiples formas de estar en la realidad (di Leonardo, 1991) y cuestiona el concepto de género en tanto que susceptible de convertirse en un concepto ahistórico y acrítico configurador de identidades esencialistas. Pero ante el reconocimiento de la inexistencia de la neutralidad del "antropólogo como autor" se plantea la necesidad de contextualizar los datos que se producen durante la observación participante ofreciendo información sobre quién los produce posición dentro de la estructura social- y con qué propósitos, así como la intersubjetividad y el diálogo en la construcción del "otro" como forma de compromiso político y ético.

Desde estos presupuestos se señala la importancia de llevar a cabo un análisis reflexivo, como un proceso de comprensión de nuestras identidades generizadas, de las complejidades codificadas de nuestro ser en el proceso de interacción en el campo. Como señala Callaway (1992), dado que las formas en que se define nuestra identidad se imbrican en la recolección de datos e interpretaciones de otras sociedades “' $[u] n$ proyecto de 'conocimiento generizado' (Caplan, 1988) requiere que el estudio de otras sociedades, incluyendo sus relaciones de género e ideologías, sea llevado con un examen escrupuloso de nosotros mismos como identidades generizadas" (Callaway, 1992: 30; traducción mía ${ }^{25}$ ).

La reflexividad planteada desde el feminismo considera al antropólogo/a en la dimensión política imbricada en las interacciones sociales en el trabajo de campo y obliga a llevar un proceso de autoconciencia en el que el género toma un lugar central. En un contexto cada vez más interconectado y desigual Callaway propone "la crítica ontológica de nosotros mismos como sujetos generizados" (1992: 45).

La crítica feminista contribuyó a romper con el dogma científico de "borrar la mancha subjetiva del trabajo de campo" (Bell, 1993) que llevaba a que la escritura etnográfica fuese despojada de lo experiencial en el encuentro con "lo otro", en pos de la búsqueda del rigor metodológico. Van Maanen (1988: 45-72) sitúa esta etnografía dentro de los "cuentos realistas"26 y guiada por la "doctrina de la inmaculada percepción" (1988: 73). La antropóloga Shepher-Hughes (1983) utiliza para referirse a este tipo de doctrina la metáfora del antropólogo como "muñeco de nieve", observando de modo penetrante pero invisible en su acción y helado en sus afectos y pasiones.

\footnotetext{
${ }^{25}$ Todas las traducciones de las obras citadas son mías

${ }^{26}$ El autor lleva a cabo una clasificación de los tipos de escritura etnográfica que se han dado en la antropología. Diferencia los "cuentos realistas", que se centran en el contenido, "Ios cuentos confesionales", más centrados en lo subjetivo, y los "cuentos impresionistas", centrados en el proceso de generación del conocimiento.
} 
Para la etnografía feminista lo emocional y lo personal no pueden ser separados de lo conceptual, como indica Okely (1975). No sólo lo personal es político, también "lo personal es teórico". El "mito del etnógrafo como héroe" imperturbable ${ }^{27}$, un dechado de virtudes que le hacía tolerante y respetuoso con las costumbres ajenas, se fue derrumbando ${ }^{28}$.

La necesidad de dar cuenta de los aspectos subjetivos y reflexivos del proceso de inmersión en las "vidas de otros" será afirmada por la antropología postmoderna. En la obra editada por Hymes en 1972, Reiventing Anthropology en el período de la "crisis del objeto" de la antropología, se enfatizará la necesidad de incorporar la reflexividad en el proceso etnográfico. Los trabajos de Clifford y Marcus (1986) y Marcus y Fischer (1986) son recurrentemente citados para situar los planteamientos de la nueva etnografía acerca de la necesidad de dar cuenta de la experiencia subjetiva que rodea al proceso de investigación etnográfica. Pero, como señala Dumont (1978), las mujeres antropólogas ya habían asumido la tarea de "conjurar la experiencia" (Callaway 1992: 37). Las mujeres acometieron "[l]a tarea de conjurar las impurezas de la experiencia: ellas hacían frente a la crueldad, al sudor y las lágrimas del trabajo de campo -sensaciones y sentimientos incluidos- mientras los hombres hacían lo que era exclusivamente cosas 'reales"' (Callaway, 1982: 37).

Como han dado cuenta diferentes autoras ${ }^{29}$ se pueden enumerar etnografías ${ }^{30}$ escritas por antropólogas que incorporan su experiencia personal y muestran su propio proceso de comprensión con sus perplejidades, descubrimientos, temores. Algunas de estas etnografías se anticiparon a las escritas posteriormente desde los presupuestos de la nueva etnografía, pero en el contexto en el que fueron escritas se consideraron más próximas al género literario que al científico ${ }^{31}$. Desde luego estos trabajos supusieron una ruptura, al mismo tiempo que su marginación al salirse de los convencionalismos que imponían borrar la subjetividad, y evitar dar cuenta de los aspectos emocionales $^{32}$. Sin embargo como ha señalado Stolcke (1996) trabajos como el de Laura Bohannan ${ }^{33}$ superan en su inquietud ética y compromiso político los realizados por los antropólogos de los 80 bajo las concepciones postmodernas (Stolcke, 1996: 340).

Muchos de estos trabajos mostraban las reflexiones y sentimientos subjetivos producidos en la interacción con lo "otro", cuestiones que no son consideradas conocimiento científico en la etnografía tradicional donde el "yo es considerado un estorbo". En el caso del trabajo de Briggs (1970) sobre los

\footnotetext{
${ }^{27}$ Véase el desarrollo de esta idea en Hayes y Hayes (1979).

${ }^{28}$ Sin duda contribuyeron hechos como la publicación del diario de campo de Malinowski (1967). Véase la polémica suscitada en Geertz (1994: 73).

${ }^{29}$ Véase Bell (1993), Callaway (1992), Okely (1992), Scheper-Hughes (1983) Stolcke (1996), entre otras.

${ }^{30}$ Ejemplos de estas etnografías son las de Abu-Lughod (1986), Bowen (1954), Briggs (1970), Fernea (1965), Myerhoff (1974, 1978), Favret-Saada (1980), Powdermaker (1967), Schrijivers (1985), Shostak (1981), Smith (1954), Suskind (1973), Wolf (1968).

${ }^{31}$ Es interesante incluir la opinión de Dumont (1978: 12) recogida en Callaway: "[n]ada me parece más ficción ahora que la clásica monografía en la que un grupo humano es representado y cuarteado a través de las categorías tradicionales de lo social, lo económico, lo religioso [...]" (1993: 39).

${ }^{32}$ Como documenta Okely (1992) algunas etnografías se publicaron bajo seudónimos (Cesarea, 1982 y Bowen, 1954$)$ y fueron consideradas "confesiones" alejadas por tanto de toda empresa científica.

${ }^{33}$ Se ocultó bajo el seudónimo E.S. Bowen porque no consideraba que escribía en este texto bajo los cánones de la disciplina (Okely 1992). Ver también los trabajos de Lutz (1990) y de Stanley (1990), citados en Bell (1990), en los que se analiza como la opción de lo experiencial y del conocedor situado en la práctica supuso la marginación de la academia, de la disciplina y del curriculum.
} 
patrones emocionales constituyó una forma de situarse en el proceso etnográfico. Otros como el de Dube $(1975)^{34}$ o el de Shostak (1981) han sido elogiados ${ }^{35}$ por la forma de mostrar la intersubjetividad en el proceso de construcción de la etnografía.

La crítica feminista va a considerar lo personal como teórico pero no va a perder el referente político del proceso de reflexividad inmerso en la práctica etnográfica. Pondrá de esta forma en evidencia cómo la Ciencia parece proveernos de un lenguaje y una estructura conceptual para el análisis de la acción política, pero no para el análisis de los sentimientos que guían nuestras acciones, alejándonos de lo que es destacado por Scheper-Hughes utilizando las palabras de Sontang (1961) de "una amplia mirada a la condición humana, elaborada con total sensibilidad" (Scheper-Hughes 1983: 115). Lo que parece mostrarse como una característica más del pensamiento occidental escindido entre razón y emoción.

Trabajos como los compilados por el Personal Narratives Group (1989), o los contenidos en Bell, Caplan y Karim (1993) o el de Schrijvers (1985) muestran la identidad generizada en el campo, fluida, abierta al cambio y al autoanálisis. La etnografía de Scheper-Hughes (1997) se inclina por el más profundo compromiso ético y político en la construcción de la alteridad, cuya escritura etnográfica aparece como "una clase especial de autobiografía intelectual, un documento personal profundo" (Scheper-Hughes, 1983). Entre las propuestas de la etnografía feministas se incluye también el género autobiográfico ${ }^{36}$ como una grabación de la experiencia del encuentro político en su contexto histórico (Okely, 1992).

En definitiva, la noción de experiencia o subjetividad que propone la perspectiva feminista poco tiene que ver con la planteada por la etnografía postmoderna. La antropología feminista, frente a la fragmentación y complejidad del sujeto postmoderno, encuentra en los procesos políticos de diferenciación el centro de su análisis, de forma que para ella la "reflexividad que excluye lo político es en sí misma irreflexiva" (Okely, 1992: 4). La experiencia etnográfica aparece como un acto total que implica cuerpo, mente, razón y emoción de manera indisoluble, como una práctica de reconocimiento de la configuración de las relaciones de poder. Por ello, sus críticas a la etnografía postmoderna se dirigen tanto a evidenciar el ocultamiento de la generización producida en el encuentro con el "otro", como a cuestionar el vacío político y el peligro relativista que implica la fragmentación del sujeto postmoderno.

\section{Bibliografía}

Ardener, Edwin (1975a). Belief and the problem of women. En Perceiving Women. Shirley Ardener, Ed. London: Dent.

\footnotetext{
${ }^{34}$ Bell, Caplan y Karim (1993) dedican su compilación a Leela Dube de quien dicen fue una de las primeras antropólogas en considerar seriamente la relación entre el género y la etnografía.

${ }^{35}$ Véase Callaway (1992) y Okely (1993).

${ }^{36}$ Véase Callan (1975), Okely y Callaway (1992), Okely (1978).
} 
Ardener, Edwin (1975b). The problem revisited. En Perceiving Women. Shirley Ardener, Ed. London: Dent.

Ardener, Shirley (1984). Gender Orientations in Fieldwork. En Ethnographic Research. R. Allen, Ed. London: Academic Press.

Asad, T., Ed. (1973). Anthropology and the Colonial Encounter. London: Ithaca Press.

Atkinson Monning, Jane (1982). Review essay: anthropology. Signs, 8(2): 236-258

Ballarín Domingo, Pilar, Gallego Méndez, Ma Teresa y Martínez Benlloch (1995). Los estudios de las Mujeres en las Universidades españolas 1975-1991. Madrid: Instituto de la Mujer.

Bell, Diane, Caplan, Pat y Wazir Jahan, Karim, Eds. (1993). Gendered fields. Women, Men y Etnography. London: Routledge.

Bell, Diane (1993). Introduction 1: The context. En Gendered fields. Women, Men y Etnography. Diane Bell, Pat Caplan y Karim Wazir Jahan, Eds. London: Routledge.

Briggs, J. (1975). Eskimo women: makers of men. En Many Sisters: Women in Cross-Cultural Perspectives. C.J. Matthiasson, Ed. New York: Free Press.

Callaway, Helen (1992). Ethnography and experience. Gender implications in fieldwork and texts. En Anthropology y Autobiography. Judith Okely y Helen Callaway, Eds. London. Routledge

Clifford, James y Marcus, George, Eds. (1986). Writing Culture: The Poetics and Politics of Ethnography. Berkeley. University of California.

De Lauretis, Teresita (1986). Feminist Studies/Critical Studies: Issues, Terms, and Contexts. En Feminist Studies/Critical Studies. T. de Lauretis, Ed. Bloomington: Indiana University Press.

Del Valle, Teresa, Ed. (1993a). Gendered Anthropology. London: Routledge.

Del Valle, Teresa (1993b). Introduction. En Gendered Anthropology. Teresa del Valle, Ed. London: Routledge.

Del Valle, Teresa (2000a). Perspectivas Feministas desde la antropología social. Barcelona: Ariel.

Del Valle, Teresa (2000b) Introducción. En Perspectivas Feministas desde la antropología social. Teresa del Valle. Ed. Barcelona: Ariel.

Di Leonardo, Micaela (1979). Methodology and the misinterpretation of women's status: A case study of Goodenough an the definition of marriage. American Ethnologist, 6 (4): 627-736.

Di Leonardo, Micaela, Ed. (1991). Gender at the crossroads of Knowledge. Feminist Anthropology in the Postmodern Era. Berkeley: University of California Press.

(C) Carmen Gregorio Gil. Publicado en AIBR. Revista de Antropología Iberoamericana, Ed. Electrónica 
Di Leonardo, Micaela (1991). Introduction: Gender, Culture, and Political Economy. Feminist Anthropology in Historical Perspective. En Gender at the crossroads of Knowledge. Feminist Anthropology in the Postmodern Era. Micaela Di Leonardo, Ed. Berkeley: University of California Press.

Flax, Jane (1987). Postmodernism and gender. Relation in Feminist Theory. Signs, 12 (4): 621-643

Golde, Peggy (1986) [1970]. Women in the Field. Berkeley: University of California Press.

Gordon, Deborah (1988). Writing culture, writing feminism: the poetics and politics of experimental ethnography. Inscriptions, 3/4: 7-24.

Gregory, J.R. (1984). The Myth of the male ethnographer and the woman's world. American Anthropology, 86: 316-27

Hacking, Ian (1983). Representing and Intervening: Introductory Topics in the Philosophy of Natural Science. Cambridge: Cambridge University Press

Haraway, Dona (1991). Ciencia, cyborgs y mujeres. Madrid. Cátedra Feminismos.

Harding, Sandra y O'Barr, Jean (1987). Sex and Scientific Inquiry. Chicago: University of Chicago Press.

Huizer, Gerrir y Mannheim, Bruce, Eds. (1979). The Politics of Anthropology: From Colonialism and Sexism Toward a View from Below. The Hague: Mounton.

Hymes, D., Ed. (1972). Reinventing Anthropology. New York: Pantheon Books.

Juliano, Ma Dolores (1990). Aportes metodológicos para los estudios de la mujer. Ponencia presentada al $V$ Congreso de Antropología, Diciembre, Granada (España).

Juliano, Ma Dolores (2000). Elaboraciones feministas y subcultura de las mujeres. En Perspectivas feministas desde la antropología social. Teresa del Valle, Ed. Barcelona: Ariel.

Kaberry, Phyllis (1939). Aboriginal Women: sacred and Profane. Londres: Routledge and Keagan Paul.

Kaberry, Phyllis (1952). Women of the Grasslands. London: HMSO. Colonial Research Publication, № 4

Fox Keller, Evelyn (1989) [1985]. Reflexiones sobre género y ciencia. Valencia: Alfons el Magnànim.

Hayes, E. N. y Hayes, Y., Eds. (1970). Claude Lévi.Strauss: the Anthropologist as Hero. Cambridge: MIT Press

Kessing, R.M. (1985). Kwaio women speak: the micropolitics of autobiography in a Solomon Island society. American Anthropology, 87: 27-39.

Landes, Ruth (1971) [1938]. The Ojibwa Woman. New York: Columbia University Press. 
Landes, Ruth (1979) [1947]. Women, Development, and Anthropological Facts and Fictions. En The Politics of Anthropology: From Colonialism and Sexism Toward a View from Below. Gerrir Huizer y Bruce Mannheim, Eds. The Hague: Mounton.

Laslett, Barbara (1990). Unfeeling Knowledge: Emotion and Objetivity in the History of Sociology. Sociological Forum, 5: 413-33.

Laslett, Barbara, Gregory K, Sally, Longino, Helen y Hammonds, Evelyn (1996). Introduction. En Gender and Scientific Authority. Barbara Laslett, Sally Gregory K, Helen Longino y Evelynn Hammonds, Eds. Chicago: The University Chicago Press.

Leith-Ross, S. (1965) [1939]. African Women: A Study of the Ibo of Nigeria. London: Routledge y Keagan Paul

López Coria, Miguel (1991). La influencia de la ecuación personal en la investigación antropológica o la mirada interior. En Los españoles vistos por los antropólogos. María Cátedra, Ed. Madrid. Júcar Universidad.

Malinowski, Bronislaw (1989) [1967]. Diario de campo en Melanesia. Madrid: Júcar Universidad

Maquieira D’Angelo, Virginia, Gómez-Ferrer Morant, Guadalupe, Ortega López, Margarita (1989). Prólogo. En Mujeres y hombres en la formación del pensamiento occidental. Vol II. Virginia Maquieira D'Angelo, Guadalupe Gómez-Ferrer Morant, Margarita Ortega López, Eds. Madrid: Ediciones de la UAM.

Marcus George y Fischer, Michael (1986). Anthropology as Cultural Critique. Chicago: University of Chicago Press.

Mascia-Lees, F.E; Sharpe, P. y Ballerino Cohen (1989). The posmodernist turn in anthropology: cautions form a feminist perspective. Signs, 15(1): 7-33.

Mathieu, Nicole Claude (1978). Man-Culture, Women-Nature?. Women's Studies International Quarterly 1(1): 5565.

Merchat, Carolyn (1980). The Death of Nature: Women, Ecology, and the Scientific Revolution. San Francisco: Harper y Row.

Morgen, Sandra, Ed. (1989). Gender and Anthropology. Critical Review for Research and Teaching. Washington: American Anthropological Association.

Moore, Henrietta L. (1991) [1988]. Antropología y feminismo. Madrid: Cátedra.

Mukhopadhyay, Carol C. y Higgins Patricia, J. (1988). Anthropological studies of women's revisited: 1977-1987. Annual Review of Anthropology, 17: 461-495.

Okely, Judith (1975). The Self and Scientism. Journal of the Anthropology Society of Oxford, 6 (3):171-88. 
Okely, Judith (1978). Privileged, Schooled and Finished: Boarding Education for Girls. En Defining Females. S. Ardener, Ed. London: Croom Helm.

Okely, Judith (1992). Anthropology and autobiography: participatory experience and embodied knowledge. En Anthropology y Autobiography. Judith Okely y Helen Callaway, Eds. London: Routledge

Okely, Judith y Callaway, Helen, Eds. (1992). Anthropology y Autobiography. London: Routledge

Ortiz, Teresa (1999). Feminismo, ciencias naturales biomédicas: debates, encuentros, desencuentros. En Cambiando el conocimiento: universidad, sociedad y feminismo. Isabel Carrera Suárez, Rosa Cid López y Amparo Pedregal Rodríguez, Eds. Oviedo: Ediciones KRK.

Personal Narratives Group, Eds. (1989). Interpreting Women's Lives. Bloomington: University of Indiana Press

Provansal, Danielle (1999). La construcción del 'nosotras' o cómo las mujeres magrebíes se piensan y se representan a sí mismas. En Actas del VIII Congreso de Antropología. Antropología del género. Carmen Díez Mintegui y $\mathrm{M}^{\mathrm{a}}$ Luz Esteban, Coord. Santiago de Compostela: FAAEE y Asociación Galega de Antropoloxía.

Rohrlich-Leavitt, Ruby; Sykes, Barbara y Weatherford, Elizabeth (1975). Aboriginal women: Male and Female Anthropological Perspectives". En Toward an Anthropology of Women. Rayna Reiter, Ed. New York: Monthly Review Press.

Saldmon, A. (1985). Maori epistemologies. En Reason and Morality. J. Overing, Ed. London: Tavistock.

Scheper-Hughes, Nancy(1983). The Problem of Bias in Androcentric and Feminist Anthropology. Women's Studies, 10:109-116.

Scheper-Hughes, Nancy (1997). La muerte sin Ilanto. Violencia y vida cotidiana en Brasil. Barcelona: Ariel.

Schirijvers, Joke (1979). Viricentrism and Anthropology. En The Politics of Anthropology: From Colonialism and Sexism Toward a View from Below. Gerrit Huizer y Bruce Mannheim, Eds. The Hague: Mounton.

Schirijvers, Joke (1985). Mothers for Life. Delft: Eburon.

Shapiro, Judith (1981). Anthropology and the study of gender. En A Feminist Perspective in the Academy. E. Langland y W. Gove, Eds. Chicago: University of Chicago.

Stacey, Judith y Thorne, Barrie (1985). The missing feminist revolution in sociology. Social Problems, 34(4):301

Stocking, George V. (1987). La antropología, ayer y hoy: Reflexiones sobre la 'crisis' y la 'reinvención' de la antropología. Conferencia presentada en el IV Congreso de Antropología, Alicante (España). 
Stolcke, Verena (1993). De padres, filiaciones y malas memorias ¿qué historias de qué antropologías?. En Después de Malinowski. Joan Bestard i Camps, Coord. Tenerife: FAAEE y Asociación Canaria de Antropología.

Stolcke, Verena (1996). Antropología del género. El cómo y el porqué de las mujeres. En Ensayos de Antropología cultural. Joan Prat y Ángel Martínez, Eds. Barcelona: Ariel.

Strathern, Marilyn (1981). Culture in a netbag: the manufacture of a subdiscipline in anthropology. Man, 16(4): 665-88.

Strathern, Marilyn (1987a). An awkward relationship. The case of feminism and anthropology. Signs, 12(2): 27692.

Strathern, Marilyn, Ed. (1987b). Dealing with inequality: analysing gender relations in Melanesia and beyond. Cambridge: Cambridge University Press.

Strathern, Marilyn (1987c). Introduction. En Dealing with inequality: analysing gender relations in Melanesia and beyond. Marilyn Strathern, Ed. Cambridge: Cambridge University Press.

Strathern, Marilyn (1998). Fuera de contexto. Las ficciones persuasivas de la antropología. En El surgimiento de la antropología posmoderna. Carlos Reynoso, Comp. Barcelona. Gedisa.

Talbot, Amaury D. (1968) [1915]. Women's Mysteries of Primitive People. The Ibibios of Southern Nigeria. London: Frank Cass.

Underhill, R. (1936). Autobiography of a Papago Woman. Memoirs of the American Anthropological Association, $N^{\circ}$ 47. Washington, D.C.: American Anthropological Association.

Van Maanen, John (1988). Tales of the Field. On Writing Ethnography. Chicago: The University of Chicago Press.

Warren, C. (1984). Gender Orientations in Fieldwork. En Ethnographic Research. R. Allen, Ed. London: Academic Press.

Weiner, Annette (1976). Women of Value, Men of Renow. Austin: University of Texas Press

Whitehead, Tony Larry y Conaway, May Ellen, Eds. (1986). Self, Sex and Gender in Cross-Cultural Fieldwork. Chicago: University of Illinois Press.

Wolf, Eric (1982). Europa y la gente sin historia. México: Fondo de Cultura Económica.

Wolfran, S (1985). Facts and theories: saying and believing. En Reason and Morality. J. Overing, Ed.London. Tavistock. 


\section{Preguntas de la editora}

Su artículo reivindica las contribuciones de la antropología de orientación feminista a los problemas epistemológicos de representación dentro de la disciplina. Señala cómo dichas aportaciones han sido rechazadas o infravaloradas desde la corriente postmoderna, a la par que muestra explícitamente las diferencias y críticas que desde la antropología de orientación feminista se realizan a los planteamientos postmodernos. ¿Podría señalar brevemente algunas de las aportaciones que la antropología de orientación feminista ha realizado al feminismo en general, y qué pueden diferenciarla de las orientaciones feministas desde otras disciplinas?

Entre las aportaciones fundamentales de la antropología social al feminismo podemos señalar la de evidenciar, mediante la riqueza de sus datos etnográficos, el carácter cultural de las diferencias de género, contribuyendo con ello a la desnaturalización de las mismas. Desde Margaret Mead, considerada por algunas autoras como prefeminista, hasta el momento actual han sido fundamentales las aportaciones teóricas y etnográficas de la antropología feminista en la comprensión y desnaturalización de las desigualdades, lo que a su vez ha planteado nuevos retos al feminismo.

La tensión entre las nociones de semejanza y diferencia ocupan para el feminismo y para la antropología un lugar central. La antropología feminista al mostrar las múltiples formas de expresión de las diferencias de género en su imbricación con otras diferenciaciones: de parentesco, sexualidad, extranjería, etnicidad, ha contribuido a la deconstrucción del sujeto mujer como categoría homogénea y esto en ocasiones se ve confrontado con la necesidad del feminismo de construir un sujeto político. La operación deconstructivista de la antropología nos lleva a dilemas del tipo ¿se puede asumir acríticamente la comunalidad de la categoría "mujer" basada en la experiencia vivida del cuerpo femenino o de un legado histórico común? O ¿cómo reconocer la heterogeneidad de la categoría mujer sin caer en la fragmentación del sujeto postmoderno? La antropología feminista no puede asentar tal sujeto político sobre bases naturales o psicológico-culturales, sin embargo sigue reconociéndose en la práctica y la ética feminista, al centrar su análisis en el poder que se encarna en cuerpos socialmente construidos como masculinos o femeninos. $Y$ en su análisis de los entresijos del poder ha contribuido a dotar de mayor complejidad a categorías centrales para el feminismo como la de patriarcado, así como a reconocer las múltiples formas de enfrentamiento al poder desde los sectores situados en posición de subalteridad.

Para terminar, creo que la antropología a diferencia de otras disciplinas tiene el potencial de promover dentro de los movimientos feministas el reconocimiento de las diferentes formas de ser mujer, sin olvidar las estructuras de poder mediante las que el sujeto mujer es producido. 